\title{
APRENDIENDO CON LAS TIC: UNA EXPERIENCIA UNIVERSITARIA
}

LEARNING FROM THE ICTS: A UNIVERSITY EXPERIENCE

\author{
Volumen 11, Número Especial
}

pp. 1-29

Este número se publicó el 30 de junio de 2011

María Gabriela Regueyra Edelman

La revista está indexada en los directorios:

LATINDEX $, \underline{\text { REDALYC}}, \underline{\text { IRESIE}}, \underline{\text { CLASE}}, \underline{\text { DIALNET, DOAJ, E-REVIST@S, }}$

La revista está incluida en los sitios:

REDIE, RINACE, OEI, MAESTROTECA, PREAL, HUASCARAN, CLASCO 


\section{APRENDIENDO CON LAS TIC: UNA EXPERIENCIA UNIVERSITARIA LEARNING FROM THE ICTS: A UNIVERSITY EXPERIENCE}

\section{María Gabriela Regueyra Edelman}

Resumen: Se comparte en este artículo la experiencia del uso de las TIC en dos cursos que se imparten en la carrera de Trabajo Social de la Universidad de Costa Rica. Se detallan los recursos tecnológicos utilizados y el uso que se da a cada uno: Facebook, Blogger, Moodle, correo electrónico, se complementa la experiencia con la incorporación de las opiniones de la población estudiantil matriculada en los cursos y destacando los retos que el uso de las TIC presenta a la población docentes en particular y a la Universidad de Costa Rica en general. El artículo retoma discusión de la Sociedad del Conocimiento y la Información, y las TIC como procesos que han transformado la sociedad actual, con su incorporación tanto en el campo económico, social y laboral como en los ámbitos educativo, familiar y personal, lo que ha generado nuevas interacciones sociales y una brecha entre la población que tiene acceso a la tecnología y la que no lo tiene, situación que ha impactado fundamentalmente el ámbito educativo, donde se han ido incorporando las TIC de manera progresiva, con algunas reservas tanto de la población docente como de la población estudiantil.

Palabras clave: TIC, DOCENCIA UNIVERSITARIA, BRECHA DIGITAL, TIC EN LA ENSEÑANZA DEL TRABAJO SOCIAL.

Abstract: This article deals with the experience of using the Information and Communication Technologies (ICTs) for two courses in the Social Work Major at the University of Costa Rica. The use of technological resources such as Facebook, Blogger, Moodle and e-mail are described. The usability of this experience is complemented with opinions from students who registered in those courses, by highlighting the challenges encountered by faculty in particular and by the University of Costa Rica in general, regarding the implementation of ICTs. This article recognizes that the Community and Information-Based Knowledge and the ICTs are means for the transformation of the community itself as they are introduced in the economic, social, labor, family, personal and educational fields. Although the application of these resources is somewhat limited for both faculty and students, their use has generated innovative social interactions, has bridged a gap between the have and have-nots and has produced an impact, specifically in the educational field, where the ICTs has been introduced progressively.

Key words: ICTS AND FACULTY AT THE UNIVERSITY LEVEL, ICTS AT THE UNIVERSITY LEVEL, ACCESS TO THE ICTS, ICTS AND STUDENT POPULATION, ICTS IN THE TEACHING OF SOCIAL WORK, CMC

\footnotetext{
1 Magister en Educación, Licenciada en Trabajo Social, ambos títulos de la Universidad de Costa Rica. Investigadora en el Instituto de Investigación en Educación. Profesora Escuela de Trabajo Social de la Universidad de Costa Rica. Asumió la jefatura de la Oficina de Beca y atención socioeconómica de la Universidad de Costa Rica, en el periodo febrero 2001 a febrero-2009.
}

Dirección electrónica: gabriela.regueyra@gmail.com

Artículo recibido: 29 de noviembre, 2010

Aprobado: 16 de junio, 2011 


\section{Introducción}

La sociedad ha ido evolucionando producto del conocimiento socialmente construido y de las grandes innovaciones tecnológicas que han revolucionado el mundo y han marcado el inicio de nuevas épocas. Algunos inventos, como el uso de la rueda en la edad de piedra o la generación de energía y la creación de la máquina, marcaron cambios radicales en las sociedades.

En la actualidad, con el acelerado desarrollo de la tecnología se ha llegado a lo que se denomina la era del conocimiento, la comunicación y la información aspectos que están revolucionando el mundo, ya que su impacto llega a todas las áreas de la sociedad, al respecto, Tünnermann plantea

La humanidad, en las últimas décadas, ha entrado en un proceso acelerado de cambios, que es la fuerza mundial más relevante, y se manifiesta en todos los ámbitos del acontecer político, social, científico y cultural, de suerte que puede afirmarse que estamos viviendo el inicio de una nueva era civilizatoria; donde la educación, el conocimiento y la información juegan un papel central. Hay quienes sostienen que no se trata de una época de cambios sino de un cambio de época. "Los fines de siglo, corrobora Fernando Ainsa, aparecen inevitablemente como la "bisagra" que anuncia un cambio de época". América Latina y el Caribe, como región, no escapan a esos procesos ni a los retos que surgen de ellos. (1999, p. 61)

Las Tecnologías de la Información y de la Comunicación (TIC) $)^{2}$ han permitido superar la distancia y el tiempo, favoreciendo el acceso al conocimiento y la información de manera rápida, sin importar el lugar donde esté almacenada, ni la ubicación de las personas que las utilizan. Muchas de las actividades que hace apenas unos años se podían realizar solamente de manera presencial como gestiones bancarias, hoy se pueden ejecutar de manera virtual, lo que ha posibilitado mayor agilidad para transacciones comerciales, financieras y servicios de muy diversa índole.

\footnotetext{
${ }^{2}$ Las TIC son definidas por el Programa de la Sociedad de la Información y el Conocimiento de la Universidad de Costa Rica (PROSIC, 2006) como: “...sistemas tecnológicos mediante los cuales se recibe, manipula y procesa información, que facilita la comunicación entre dos o más interlocutores. Por lo tanto, las TIC son algo más que informática o computadoras, puesto que no funcionan como sistemas aislados, sino en conexión con otras mediante una red. También son algo más que tecnologías de emisión y difusión (como televisión y radio), puesto que no sólo dan cuenta de la divulgación de la información, sino que además permiten una comunicación interactiva" p. 8.
} 
En la vida cotidiana, el uso de la televisión permite mantenerse informado de los últimos acontecimientos a nivel mundial, una situación de emergencia al otro lado del planeta se puede presenciar en tiempo real ${ }^{3}$. La comunicación en tiempo real y diferido con voz e imagen en computadora o teléfono acerca a las personas sin que la distancia o la ubicación sea una limitante.

En los procesos educativos la incorporación de recursos audiovisuales, como presentaciones utilizando multimedia, videos, conferencias, películas en el caso experiencias presenciales o las aulas virtuales, recursos en la Internet en procesos de aprendizaje virtuales, así como el acceso virtual a bibliotecas, revista y libros ha generado características distintas en los actuales procesos educativos.

El avance de la sociedad ha sido producto del conocimiento del ser humano, de ahí que para lograr el desarrollo económico y social de un país, se debe socializar el conocimiento generado, promover la investigación científica y la innovación, así como el uso de tecnologías de punta, lo que le permitirá no solo alcanzar niveles de desarrollo económico, sino también el bienestar de su población

Existe un amplio reconocimiento de la importancia que tienen los avances científicos y el cambio tecnológico como medio para lograr mejores niveles de desarrollo económico y bienestar de los ciudadanos. Es así como las economías del conocimiento sustentan su éxito en la capacidad para crear, distribuir y explotar el conocimiento. Los aspectos relacionados con la incorporación del conocimiento a la actividad humana están ampliamente reconocidos, hay estudios económicos, y en otras áreas, que demuestran la relevancia y pertinencia de invertir en el desarrollo de la ciencia, la tecnología y la innovación. No hay duda, de que este proceso se ha visto acelerado y transformado por el gran impacto de las TIC sobre la economía y la sociedad (PROSIC, 2006, p.3). (...) El desarrollo económico, político y social de un país se determina en gran medida por su avance científico, tecnológico y de innovación. Bajo esta perspectiva, los avances en las Tecnologías de Información y Comunicación (TIC), incluyendo la microelectrónica, aunados a la globalización de la economía y al incremento en los flujos de capitales y

3 Ejemplo de esto fue el rescate de los mineros chilenos en octubre del 2010, que fue visto en tiempo real desde diferentes países del mundo. 
mercancías, imponen grandes retos a los países para evolucionar hacia una economía basada en el conocimiento. (PROSIC, 2006, p.3).

La Sociedad de la Información y las Tecnologías de Información y Comunicación (TIC) surgen acompañadas de preocupaciones, sobre la participación del ser humano en estos procesos de construcción y divulgación del conocimiento, así como del acceso a las TIC. Establecen como uno de los principales retos, colocar a la persona en el centro de estos desarrollos, superando paradigmas de otras visiones de mundo (Mato, Santamaría, Silva, y Cheaz, 2001, p. 19) que centran el desarrollo en la máquina (herencia de la era industrial) o en el mercado (base del sistema capitalista globalizado), lo que se evidencia en la declaración de principios de la Cumbre Mundial sobre la Sociedad de la Información, realizada por el Consejo de la Unión Internacional sobre las Telecomunicaciones (UIT), en el año 2001 en Ginebra, primera parte, y en el 2005 en Túnez, segunda parte, donde

...se declaró el deseo y compromiso común de construir una Sociedad de la Información, centrada en la persona, inclusiva y orientada al desarrollo, en la que todos podamos crear, acceder, utilizar y compartir información y conocimiento, permitiendo a individuos y comunidades lograr su potencial, y mejorar su calidad de vida. (p. 21)

Se declararon, además, los siguientes principios:

- Mejorar acceso a la información y el conocimiento.

- Construir capacidad de personas y empresas.

- Aumentar la confianza y seguridad en el uso de las TIC.

- Crear un ambiente propicio.

- Desarrollar y ampliar las aplicaciones de las TIC.

- Promover y respetar diversidad cultural.

- Reconocer el papel de los medios.

- Reconocer la dimensión ética de la SIC.

- Promover cooperación internacional y regional.

Se indica, asimismo, que es esencial la participación del Estado, la empresa privada, la sociedad civil y los organismos internacionales, en el desarrollo de la Sociedad de la información con acceso universal, ubicuo, equitativo y accesible a la infraestructura y servicios de las TIC, adaptado a las condiciones regionales, nacionales y locales (PROSIC, 2006, p. 21). 
Partiendo de esos principios, el concepto de Sociedad de la Información da: "un énfasis especial en la forma en que las sociedades pueden utilizar o utilizan las TIC para alcanzar mayores niveles de desarrollo y de calidad de vida" (PROSIC, 2006, p. 8), tal como quedó ratificado en la Cumbre Mundial.

Jeskanen-Sundström (2001, citado en PROSIC, 2006) define la Sociedad de la Información como:

una sociedad que hace un uso extensivo de las redes y de las tecnologías de la información y la comunicación, produce grandes cantidades de bienes y servicios ligados a la información y a las comunicaciones, y ha logrado una importante diversificación de su industria. (p. 8).

La velocidad con que se ha introducido la tecnología en los hogares, la escuela, los lugares de trabajo, en el entorno económico y social ha provocado grandes cambios en las relaciones familiares, sociales y económicas, es decir, han generado una brecha entre la población que tiene acceso a estas tecnologías y las que no la tienen, consecuencia directa de una sociedad socioeconómicamente desigual. Por un lado, las poblaciones que se ubican en extractos sociales con limitaciones económicas para satisfacer sus necesidades fundamentales, no tienen acceso a estos recursos tecnológicos y junto con las generaciones que nacieron antes de la era digital, conforman una población que se ha quedado al margen de estos desarrollos tecnológicos, a esta situación se le ha denominad brecha digital; al respecto, Periró comenta

Muchos son los estudios que intentan explicar los cambios que la introducción de las TIC's han provocado a nivel social, cultural y económico. Todos coinciden en que, al romperse las barreras del espacio y el tiempo, la sociedad se globaliza, aunque se creen nuevas fracturas como la denominada brecha digital que se refiere al problema de exclusión social sufrido por las personas que no pueden acceder o aprovechar las ventajas que ofrecen las TIC's (Periró 2000, citado por Bustos, s.f., p. 7)

\section{Las TIC en Costa Rica}

Costa Rica se ha destacado a nivel mundial por ser un país interesado en promover Políticas Educativas de Información, Ciencia y Tecnología, que han favorecido del desarrollo económico y social, tal como se plantea en el Informe del PROSIC (2007) 
Debido al estilo de desarrollo económico-social y a la visón de la educación que ha prevalecido desde la constitución de la Segunda República en 1949, Costa Rica es reconocida mundialmente como un país pionero en el desarrollo de infraestructura en telecomunicaciones en la implementación de políticas públicas asociadas a la educación, la salud, la modernización de la economía y la productividad. (PROSIC, 2007, p. 188)

La intervención gubernamental, en esta materia de acuerdo con el Informe del PROSIC, se inicia en el periodo 1990-1994 con el Programa Nacional de Informática, cuyo principal objetivo fue: "promover la utilización de la Informática como instrumento para proponer cambios en el ambiente tecnológico y cultural del país" (PROSIC, 2007, p. 6). En el periodo 1994-1998 los esfuerzos fueron orientados en: "construir las futuras autopistas de información nacionales y servicios que permitieran a los costarricenses un amplio acceso a la información" (PROSIC, 2007, p. 6).

De 1998 al 2000, los esfuerzos del gobierno se concentraron en: "el desarrollo de acciones tendientes a incrementar el acceso a las TIC, concediendo especial importancia a los factores que inciden en el costo de acceso a la Internet, en especial la infraestructura de telecomunicaciones, con el fin de abaratar dicho costo" (PROSIC, 2007, p. 7).

En el período 2002-2006, se da continuidad a la política de promoción del uso de las TIC, que queda evidenciado con la formulación del Plan de Gobierno Digital 2002-2006. En el Programa de Gobierno 2006-2010, no se propone un plan específico sobre el gobierno digital; sin embargo: "aparece mencionado en tres de las cinco áreas de acción en que se estructura dicho programa: las Políticas Sociales, las Políticas Productivas y la Reforma al Marco Legal e Institucional" (PROSIC, 2007, p. 10).

Específicamente. en el área educativa en secundaria se reconocen como las primeras iniciativas de la incorporación de las TIC, primero la Red Centro para la Enseñanza de la Informática (CEI), en el año 1985, y luego en el año 1987 con la creación de la Fundación Omar Dengo, que tenía como objetivo: "el mejoramiento de la calidad de la educación costarricense, por medio del uso de las herramientas informáticas para promover la innovación y la calidad educativa" (PROSIC, 2007, p. 188).

Para el caso de la educación superior en Costa Rica, el PROSIC coloca como uno de los principales desafíos: "impulsar y desarrollar la ciencia y a tecnología, y elevar la calidad de la 
formación profesional, ya que en el país y en la región los indicadores muestran grandes diferencias respecto a los países desarrollados" (PROSIC, 2007, p. 200).

Desde el Consejo Nacional de Rectores, ${ }^{4}$ y en el marco del debate de la conferencia Mundial sobre Educación Superior de 1998, en el año 2002 es creada la Comisión de Tecnologías de Información y Comunicación para la Educación Superior (TICES) a solicitud de la Comisión de Vicerrectores de Docencia (PROSIC, 2007, p. 200).

En el año 2003, esta comisión:

publicó un documento con sugerencias y recomendaciones para el desarrollo de la política universitaria que favorezca e incentive la integración de las TIC en los procesos de enseñanza y aprendizajes en el sistema de educación superior pública costarricense, el documento se hizo con base en un diagnóstico de las cuatro universidades púbicas sobre las políticas y acciones institucionales vinculadas a las TIC. (PROSIC, 2007, p. 20)

En el marco del PLANES 2006-2010, PROSIC plantea que

la tecnología es un componente fundamental. En particular, las TIC y su integración progresiva en las actividades de docencia, acción social e investigación se consideran indispensables para el logro de las metas del periodo 2006-20120. El desafío de las universidades públicas costarricenses es encontrar mecanismos para reactivar el desarrollo nacional y modernizar la formación de profesionales desde una sólida base científica y tecnológica. (2007, p. 203)

\section{La Universidad de Costa Rica y las TIC}

La Universidad de Costa Rica creó en la década de 1980 la Escuela de Ciencias de la Computación e Informática, tres de sus docentes, Max Cerdas, Guy de Teramond y Claudio Gutiérrez, presentaron la propuesta: "la implementación de la red académica BITNET en el país y en Centroamérica" y de "conectar a la comunidad universitaria de la región centroamericana a esa red en la Conferencias Espacial de las Américas de 1990" (PROSIC, 2007, p. 203).

Desde que inicia los primeros pasos en el uso y acceso a las TIC, la Universidad de la Costa Rica ha tenido como horizonte la visión humanista y su compromiso con la sociedad

4 Consejo Nacional de Rectores (CONARE), integrado por los rectores de las universidades púbicas del país, responsable de la formulación del Plan Nacional de la Educación superior (PLANES) (PROSIC, 2007, p. 200). 
costarricense, principios que orientan su quehacer de acuerdo con el Título I "Principios y Propósitos" del Estatuto Orgánico (1974) y que comparte, como se mencionó al inicio de este escrito, con los que se postulan en la declaración de la Cumbre Mundial sobre la Sociedad de la Información, que se pueden sintetizar en lo se plantea en el Informe del PROSIC

El primer aspecto se fundamenta en la visión humanista y solidaria, en donde la creación, la adaptación y difusión del conocimiento tenga como eje esencial al ser humano. Así, desde esa perspectiva humanista, la persona y la búsqueda de una mejor calidad de vida son los ejes esenciales que sustentan toda la actividad que generaremos para el desarrollo del conocimiento. Esto conlleva a establecer las vías pertinentes para que el conocimiento tenga un carácter universal y que la mayor cantidad de ciudadanos puedan incorporarlo a su vida diaria. (PROSIC, 2006, p. 4)

Una de las acciones concretas que evidencia el compromiso de la Universidad de Costa Rica con la sociedad costarricense es la creación, en el año 2005, del Programa de la Sociedad de la Información y el Conocimiento de la Universidad de Costa Rica (PROSIC) con el fin de ser:

un espacio multidisciplinario, con un enfoque central en el ser humano, dedicado al estudio, la reflexión, el análisis de políticas y el desarrollo de actividades y proyectos para un mayor y mejor uso de las tecnologías de información y comunicación (TIC) en beneficio de la sociedad. (PROSIC, 2006, Presentación)

Asimismo, en los últimos años ha venido incorporando, cada vez con mayor fuerza, el uso de la tecnología en sus actividades sustantivas de docencia, investigación y acción social, así como en las áreas de vida estudiantil y administración, lo que se evidencia en las Políticas Prioritarias para la Formulación y ejecución del Plan Presupuesto de la UCR para el año 2005, la promulgación de las Directrices Generales en Tecnologías de Información y comunicación (TIC's) u-digit@I-Plan de mejora 2010 (UCR-2010a) y las Políticas 2010-2014, específicamente, las que están detalladas a continuación

\subsection{Desarrollo Académico y Evaluación}

2.2.1. Impulsará mediante todas sus instancias, gestiones con criterios de calidad y flexibilidad, que le permitan interactuar, de manera solidaria, en la consecución de la excelencia académica y el mejor aprovechamiento de los recursos. 
2.2.2. Promoverá el desarrollo de un currículo innovador, creativo, flexible e inclusivo, que integre una perspectiva de derechos, de género, de protección del ambiente y el uso de nuevas tecnologías.

2.2.7. Apoyará el uso de nuevas tecnologías, para optimizar los procesos de enseñanza-aprendizaje y fortalecer la investigación y la acción social, así como para agilizar los procesos de admisión, permanencia y graduación. Universidad de Costa Rica. (UCR, 2008, p. 5.)

En esta misma línea, la Universidad de Costa Rica ha venido impulsando programas de capacitación dirigidas al personal docente ${ }^{5}$ para que construya nuevos procesos educativos acordes con la expectativas y necesidades de las nuevas generaciones, que han crecido en un ambiente donde el uso de la tecnología es cotidiano, lo que les ha permitido desarrollar nuevas habilidades y destrezas que, a su vez, genera nuevas expectativas hacia el proceso educativo; en ese sentido, en el informe del PROSIC, está planteado "Garantizar la dotación sostenible de recursos humanos calificados es uno de los pilares fundamentales de una sociedad basas en el conocimiento. Es esencial contar con una estrategia de mediano y largo plazo para la formación de recursos humanos" (2006, p. 5).

Por tal motivo, las características de las nuevas generaciones colocan a la población docente universitaria ante un gran reto, la población estudiantil maneja con mayor destreza las TIC y los conocimientos se encuentran al acceso de todos en la Internet. Estos aspectos conllevan, además de tener que desarrollar destrezas y habilidades en el uso de las TIC, replantear el proceso pedagógico y construir estrategias novedosas para la formación de los futuros profesionales.

Pero, iqué difícil para un docente que se formó en otra época!, lejos de la tecnología, la cual ha ido incorporando paulatinamente, en muchos casos con temor y hasta dificultades para

\footnotetext{
5 Algunos ejemplos, por un lado, desde el "Programa de Tecnologías Avanzadas PROTEA, de la Facultad de Educación de la Universidad de Costa Rica, se han impartido cursos como: "Educar para el futuro", dirigido al personal docente para promover el uso de las TIC en el aula, capacitación, para la elaboración de mapas conceptuales y el uso herramientas como Cmaptools, el uso de la web y de redes sociales en la docencia; por otro, la apertura, en el año 2009, de la Licenciatura en Diseño y desarrollo de espacios educativos con TIC, que se imparte en la Sede del Atlántico; además, en el Recinto de Paraíso, y desde la Vicerrectoría de Docencia, se promueve el uso de la "Plataforma de mediación virtual", recurso virtual disponible para que las y los docentes universitarios acompañen en el curso presencial con TIC y, por último, desde la Red Institucional de Formación y Evaluación docente (RIFED) esta misma Vicerrectoría continúa con diferentes iniciativas de capacitación promoviendo en el uso de las TIC en el aula.
} 
comprender su uso, desarrollar habilidades y destrezas para utilizarlas en su ejercicio como profesional y docente universitario.

Sin embargo, es importante reconocer la capacidad que tienen las personas para aprehender a largo de toda su vida, ya sea como personas, como profesionales y como docentes. Para el caso de la población docente, participar en procesos pedagógicos le favorece en la construcción de nuevo conocimiento, así como en el desarrollo de nuevas habilidades y estrategias.

Serán muchas las veces en que la población estudiantil favorezca ese crecimiento de la población docente, al retroalimentar el proceso educativo con información que ha obtenido o al resolver diferentes situaciones que se le presenten al docente al hacer uso de las TIC en el aula, como por ejemplo: un problema de acceso o conectividad de las computadoras que el docente no logró resolver en el aula, alguno o alguna de las y los estudiantes lo logra rápidamente.

Es que, como lo plantea García, las nuevas generaciones

Forman parte de una generación que ha crecido inmersa en las Nuevas Tecnologías, desarrollándose entre equipos informáticos, videoconsolas y todo tipo de artilugios digitales, convirtiéndose los teléfonos móviles, los videojuegos, Internet, el email y la mensajería instantánea en parte integral de sus vidas y en su realidad tecnológica. Navegan con fluidez; tiene habilidades en el uso del ratón, utilizan reproductores de audios y video digitales a diario; toman fotos digitales que manipulan y envían; y usan además sus ordenadores para crear videos, presentaciones multimedia, música, blogs, etc. (...) les encanta hacer varias cosas al mismo tiempo: son multitarea. Afrontan distintos canales de comunicación simultáneos, prefiriendo los formatos gráficos a los textuales. Utilizan el acceso hipertextual en vez del lineal. Funcionan mejor trabajando en red. Y prefieren los juegos al trabajo serio. (García, 2010, p. 2)

No obstante, a pesar de que existe población joven que tiene las características que plantea García, la sociedad actual, en general y nuestro país en particular, enfrenta la inequidad en el acceso de las TIC, generada por desigualdad social que caracteriza las actuales sociedades, lo que queda evidenciado en los procesos educativos cuando no toda la población estudiantil ha logrado tener acceso a las TIC. 
Por tal motivo, en una investigación realizada a las y los jóvenes costarricenses estudiantes universitarios y de segunda enseñanza en Costa Rica en el año $2007^{6}$ y publicado por el PROSIC en el año 2008 titulada: Los jóvenes costarricenses en la sociedad de la información, están planteadas, claramente, las limitaciones que enfrenta esta población para poder utilizar las TIC y entre ellas la principal es los limitados recursos económicos

Los jóvenes que se encuentran en condiciones económicas más bajas indican tener mayores problemas para utilizar las TIC que los de condiciones más altas. La falta de dinero es señalada como un obstáculo por el $76,5 \%$ de los jóvenes de nivel económico bajo, mientras que entre los de nivel alto solamente un $37 \%$ lo señalaron. El $44,7 \%$ de los jóvenes con condiciones económicas bajas indicó el acceso de Internet como impedimento para el mayor uso de las TIC, mientras que los de condiciones económicas altas lo indicaron en menos del 6\%. (PROSIC, 2008, p. 67)

En consecuencia, la Universidad de Costa Rica, siendo una universidad pública que promueve el acceso en igualdad de condiciones, ha favorecido el ingreso de población proveniente de sectores sociales con limitaciones económicas y con aptitudes para continuar los estudios universitarios, reflejado en el $52 \%$ de la población estudiantil de la Universidad de Costa Rica que disfruta de beca por condición socioeconómica y un $38 \%$ de la población becada que cuenta con la categoría de beca 11 (UCR; 2010 b), la que, además de la exoneración del $100 \%$ sobre los montos de matrícula, recibe ayuda socioeconómica. Si esta población tiene limitaciones económicas para continuar sus estudios universitarios, puede plantearse que una parte de esta población en particular tiene limitado acceso a las $\mathrm{TIC}^{7}$.

De acuerdo con el estudio realizado por el PROSIC: "La tenencia de computadoras se da en tres cuartas partes de los hogares de los jóvenes (73,9\%) y menos de la mitad (44,55\%) tienen acceso a Internet desde el hogar en población joven" (PROSIC, 2008, p. 26), lo que

6 El $62 \%$ de los jóvenes entrevistados pertenecen a colegios y el restante $37,9 \%$ pertenecen a universitarios. (PROSIC, 2008, p 11)

A pesar de que se carece de un estudio específico sobre el acceso que tiene la población becada por condición socioeconómica a las TIC, de acuerdo con la investigación del PROSIC sobre el acceso de los jóvenes estudiantes de educación secundaria y universitaria, se puede plantear como una posibilidad. Situación que se indagó con la población de dos cursos de la Escuela de Trabajo Social de la Universidad de Costa Rica y se presenta en este escrito. 
obviamente los coloca en una posición de desventaja frente a sus compañeros y compañeras que sí tienen acceso a computadoras e internet en sus hogares.

De igual modo, el caso de tener su propio celular ${ }^{8}$, que les permita estar en comunicación permanente con la población de la cual forman parte, o acceso a programas de televisión por cable que les permite tener un panorama más amplio del entorno mundial. Esto muestra lo planteado como la brecha digital introducido en las páginas iniciales de este escrito que no sólo se refiere al acceso de las diferentes generaciones a las TIC, sino también al acceso que tienen los diferentes sectores sociales que integran nuestra sociedad costarricense.

Ante esta situación, la Universidad de Costa Rica tiene como reto poner a disposición de la población estudiantil el acceso a las TIC y desde la docencia desarrollar procesos pedagógicos con una población estudiantil que no comparte el mismo desarrollo de habilidades y destrezas en el uso producto de sus limitaciones de acceso.

Las diferentes Unidades Académicas, así como otros servicios universitarios v. gr. el Sistema de Bibliotecas, Documentación e Información SIBSI, ponen a disposición de la población estudiantil equipos de cómputo con acceso a la Internet; sin embargo, la demanda de éstos equipos supera la oferta disponible, los horarios para su uso es limitado y las características de los mismos no satisfacen todos los requerimientos de la población estudiantil; por eso, en el estudio realizado por el PROSIC, 2008, se plantea que la población estudiantil entrevistada considera que "las universidades deben mejorar la calidad en equipo tecnológico como computadoras (73\%), impresoras (72,3\%) y proyectores multimedia $(69,5 \%)$ (PROSIC, 2008, p. 60).

En este contexto universitario, docentes de diversas áreas realizan experiencias innovadoras, enfrentando los retos que el uso de TIC genera en el proceso pedagógico en los cursos que imparten en la Universidad de Costa Rica; en esta línea, queda compartida la experiencia que he desarrollado con dos cursos en la carrera de Trabajo Social de la Universidad de Costa Rica.

8 El $80 \%$ de los jóvenes tienen teléfono residencial y un $80,6 \%$ celular. 


\section{Experiencia con el uso de las TIC en dos cursos universitarios ${ }^{9}$}

Antes del inicio del curso lectivo, la población docente debe elaborar el Plan de curso de acuerdo con las líneas curriculares y el plan de estudios de la carrera. La propuesta general luego se va detallando en la planificación de cada clase, incorporando contenidos que retomen los conocimientos, habilidades y destrezas que se deben construir y desarrollar con la población estudiantil.

En este proceso de reflexión y planificación es cuando la o el docente determina la metodología y las técnicas que utilizará para lograr alcanzar los objetivos del curso. Las TIC son un recurso para alcanzar los objetivos de conocimiento destrezas y aptitudes.

La experiencia con el uso de las TIC en los cursos fue construyéndose progresivamente en etapas, conforme la docente iba aprendiendo el uso de los diferentes recursos y participado en procesos de capacitación que favoreciera el desarrollo de habilidades y destrezas.

\section{Primera etapa}

Desde mi experiencia, primero utilicé los recursos audiovisuales en las clases presenciales como videos, películas y presentaciones con software como el Power Point $\circledast$ y Cmaptools.

El utilizar videos y películas requirió de previo la búsqueda de producciones (videos y películas) y valorar los contenidos y su relación con los temas propuestos en el curso, así como la forma en la que se iban a utilizar: introducir el tema, generar discusión grupal, reflexionar de manera individual, organizar el conocimiento adquirido desde estos recursos sintetizándolo en reportes, entre otros.

En un principio, para el desarrollo de los temas del curso, la docente debe ordenar la secuencia de los contenidos con un orden y estructura lógica, el uso de diagramas, esquemas e imágenes utilizando herramientas como el Power Point ${ }^{\circledR}$, pueden contribuir a favorecer la atención y el aprendizaje de la población estudiantil, ya que junto a la exposición de la docente se reafirmaron contenidos de manera visual. Todo ello es el resultado de un esfuerzo previo de investigación, comprensión, organización, desarrollo de conocimiento y construcción de

9 Carrera de Trabajo Social de la Universidad de Costa Rica: TS-5118: Ideología Ética y Derechos Humanos III y TS. 4018: Fundamentos teórico metodológicos del trabajo social VI: Fenómeno de las drogas. 
esquemas visuales por parte de la docente, que logra sintetizar con la tecnología en un recurso visual.

Después, para lograr favorecer el desarrollo de habilidades y destrezas de: investigación, comprensión, organización, desarrollo de ideas y construcción de esquemas visuales que facilita la reconstrucción del conocimiento adquirido desde sus propios esquemas mentales, debe promoverse en la población estudiantil el uso de herramientas tecnológicas y el acceso a la Web.

Desde ésta última acercarse al conocimiento acumulado y con la utilización de otros recursos, como las presentaciones en Power Point $\AA$, recrear organizar el conocimiento adquirido al tener que procesarlo para elaborar la presentación y exponer ante sus compañeros o compañeras de clases

En síntesis, con el uso de estos recursos tecnológicos en los procesos pedagógicos se logra un estímulo auditivo y visual que propicia el aprendizaje y la reconstrucción del conocimiento, desarrollando nuevas experiencias como docente; en relación con este tema Bustos plantea

En la Sociedad del conocimiento caracterizada por las sobre abundancia de información, se pone en el centro del proceso al estudiante y se propicia en él el desarrollo de las competencias de análisis, evaluación y síntesis de la información, de pensamiento estratégico, de forma que cree y genere nuevo conocimiento. El Profesor se convierte en un mediador de contenidos y en guía del aprendizaje de cada estudiante, individualizando su actuación. (Bustos, s.f., p. 12)

Para el uso de los recursos mencionados, en los cursos de Trabajo Social, fue necesario realizar coordinaciones para reservar salas de audiovisuales, solicitar, trasladar e instalar el equipo $^{10}$. El proceso de incorporación de estos recursos en el aula se enfrentó a limitaciones externas a la docente como fueron: la disponibilidad y las características de los equipos, las

\footnotetext{
10 Aunque parezca fácil muchas veces no resulta como estaba planeado: el equipo no prende o no se logra la conexión entre el CPU y el equipo de proyección, hay mucha iluminación en aula y no se pude oscurecer, los conectores de electricidad no sirven en algunas aulas, no se cuenta con adaptadores de tres a dos, ya que en los edificios hay tomacorrientes de dos entradas y los equipos requieren tres, no se cuentan con extensiones, no hay pantallas, los equipos de cómputo son pesados para ser trasladados a las aulas, todo ello se convierte en factores que atrasan el inicio de la lección y genera angustia en la o el docente.
} 
condiciones físicas y tecnológicas de los edificios que se construyeron en una época cuando la herramienta en la docencia era la pizarra (primero de tiza y luego de marcadores).

\section{Segunda etapa}

Nuevos retos empiezan a surgir cuando la población estudiantil solicita tener acceso de manera digital a los recursos audiovisuales utilizados, como las películas, los videos, las exposiciones o demandan comunicaciones vía correo electrónico u observaciones a material de consulta o textos electrónicos, o a comunicarse por la web con los docentes, medios de interacción social que utilizan cotidianamente para comunicarse con sus iguales y, en muchos casos, utilizados en procesos de enseñanza en la educación secundaria y universitaria.

Esta situación lleva a trascender el proceso de enseñanza aprendizaje y la interacción de la o el docente con la población estudiantil en el espacio tradicional del aula (lugar concreto) y en tiempo real, a un espacio virtual y un tiempo diferido y, con ello, se transforma el espacio pedagógico y proceso que las y los docentes conocemos, ya que las TIC conllevan una nuevas dimensiones; en tales términos, Area comenta

Uno de los efectos más notables de las tecnologías digitales es que permiten y facilitan una mayor comunicación entre las personas independientemente de su situación geográfica o temporal. Las nuevas tecnologías de la comunicación rompen barreras espacio-temporales facilitando la interacción entre personas mediante formas orales (telefonía), escrita (el correo electrónico) o audiovisual (la video conferencia). Asimismo esta comunicación puede ser sincrónica-es decir, simultánea en el tiempo- o asincrónicael mensaje se emite y recibe en un periodo de tiempo posterior al emitido. En segundo lugar, podemos señalar que las tecnologías permiten el acceso de forma permanente a gran cantidad de información. (Area, 2009, p. 7)

Con todo, la Universidad de Costa Rica tiene a disposición de la docencia universitaria una plataforma MOODLE ${ }^{11}$ para la mediación de la docencia con Tecnologías de la Información y Comunicación, que contiene espacios para aulas virtuales que apoyan los cursos presenciales, con diversas actividades y la utilización de herramientas de comunicación, permite entre otros: organizar el curso, colocar materiales digitales (películas, presentaciones en Power

11 Dirección electrónica: www. mediaciónvirtual.ucr.ac.cr 
Point $\AA$, lecturas), tareas o actividades en línea, foros, wikis, conexión con sitios de interés para el curso, mensajería para la población inscrita entre otros, esta plataforma es administrada por la Unidad de Apoyo a la Docencia Mediada con Tecnologías de la Información y la Comunicación (METICS) ${ }^{12}$, de la Vicerrectoría de Docencia. El utilizar la plataforma administrada por METICS fue mi segunda experiencia.

Luego de recibir un curso de inducción sobre el uso esa plataforma, preparé el curso de manera bimodal, utilizando clases presenciales y actividades en el aula virtual. El proceso de preparación del curso previo al inicio de cada ciclo lectivo fue todo un reto, debía diseñar el aula virtual y preparar las lecciones presenciales. El utilizar mapas conceptuales para organizar los contenidos, las secuencias y los recursos fue de gran ayuda para integrar la modalidad presencial y virtual.

El aula virtual colocada en la plataforma es un apoyo al curso, lo que implica que deben prepararse contenidos, materiales y recursos de apoyo, tanto para las clases presenciales, como para el aula virtual, así como el tener claridad sobre la estrategia metodológica para el desarrollo de las habilidades y de las destrezas planteadas en el programa del curso.

La primera vez se necesita de más tiempo, ya que debe enfrentar se el proceso de desarrollo de habilidades y destrezas en el uso de un nuevo software, lo que genera mucha ansiedad y requiere de mayor esfuerzo. En este proceso ha sido muy importante el apoyo recibido por el equipo de profesionales responsables de METICS, que vía telefónica o presencial ofrecen asesoría permanente a los docentes.

Así pues, había organizado el espacio del curso por semana, cumpliendo con las indicaciones en el uso de estas herramientas; elaboré una presentación del curso, coloqué fotografías alusivas al tema tratando de hacer agradable el ingreso a la página. Desde el primer día les coloqué material para acompañar el curso, indicaciones, material en Word, exposiciones de clases con ayuda de Powerd Point $\AA^{\circledR}$.

Como parte de las actividades, fueron programados dos foros: uno en el laboratorio de cómputo de la escuela y otro desde los lugares que cada estudiante escogiera para la actividad. En el primer foro, que para mí también era la primera experiencia como docente, me acompañó

12 Creada en el año 2006. Tiene como propósito apoyar a la comunidad docente universitaria en: A) Promoción y desarrollo de entornos educativos basados en el uso de TICS. B) Desarrollo y socialización de experiencias e innovaciones pedagógicas apoyadas con TICS. C) Apoyo a proyectos académicos que requieren de alto grado de autonomía. D) Capacitación para el desarrollo de la docencia mediada con TICS. 
un funcionario de Mediación Virtual, quien motivó a la población estudiantil sobre el uso de la plataforma y los foros junto al cual aprendí el uso de este recurso. Esta primera experiencia la realicé en el laboratorio de cómputo de la Unidad Académica, fue agradable para todos.

El aprendizaje me permitió manejar con mayor seguridad el recurso, la segunda experiencia la realicé desde mi casa, monitoreando y acompañando a la población estudiantil. En ambos momentos fue cumplido con el objetivo propuesto, la población estudiantil logró sintetizar y reconstruir de manera individual y colectiva, el conocimiento adquirido sobre uno de los temas del curso ${ }^{13}$.

Dos cursos he logrado construir en la plataforma de mediación virtual: uno organizado por sesiones semanales y otro por unidades Temáticas, que se puede apreciar en la siguiente imagen

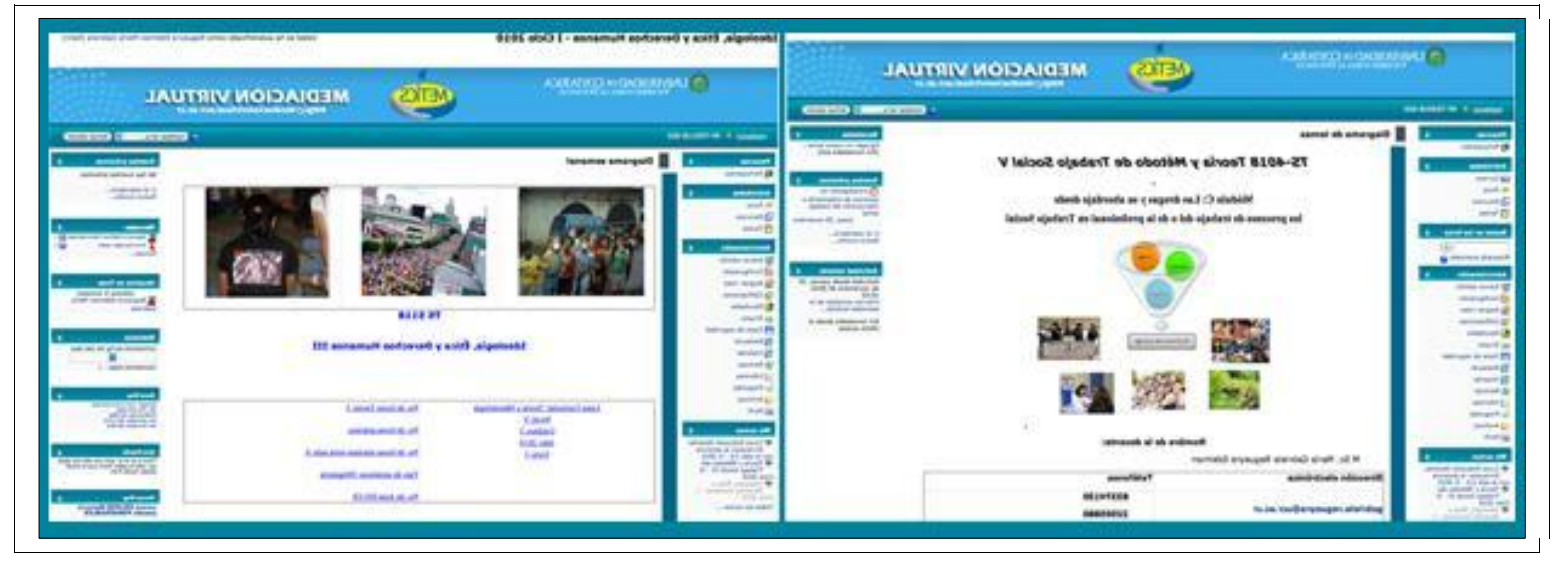

\section{Tercera etapa}

Los retos continuaron, necesitaba nuevos espacios de apoyo que facilitaran el intercambio con la población estudiantil de una manera ágil e informal, que favorecieran la discusión y respondieran consultas cortas sobre asignaciones específicas, lo que me llevó a valorar otras opciones de la web como son las redes sociales y el blog.

A pesar de mis reservas hacia el uso de redes sociales, tanto en el área personal como en la docencia y de recibir capacitación sobre su uso, lo consideré como un posible recurso en

\footnotetext{
13 Sin embargo, es necesario aclarar que he enfrentado ciclos lectivos en los cuales para poder realizar un foro en tiempo real ha sido necesario reservar un laboratorio de cómputo, ya que no toda la población estudiantil, como se ha venido comentando, cuenta con un lugar apropiado para realizarlo, pues no tienen los recursos en sus hogares
} 
mi labor docente. Aprendí que era una herramienta que favorecía la comunicación, el intercambio y la socialización, así como una de los recursos de la web, más utilizada entre la población joven.

El uso del Facebook® permitió evacuar consultas sobre trabajos específicos a la población estudiantil, cada vez que colocaba algo en el llamado "muro" como respuesta a una consulta, la población estudiantil se enteraba de que se había colocado nueva información, así se iba construyendo, automáticamente, una bitácora con todas las preguntas y respuestas, encontré frases que decían: "profesora le iba a hacer una pregunta, pero ya vi la respuesta". Las preguntas las escribían en el muro, por mi parte, revisaba de manera frecuente el perfil para dar respuesta, la población estudiantil sabía que ahí la encontraría.

Mientras tanto, la apertura de un blog ${ }^{14}$ facilitó la creación de un espacio informal de discusión y de actualización sobre la temática del curso, la colocación de material específico y vínculos con diversos recursos como noticias nacionales e internacionales, videos, películas libros etc., con espacios para comentar y reaccionar ante dichos materiales le dio una dimensión amplia que trascendió los contenidos programados y amplió el panorama de la población estudiantil, la libertad de acción de cada estudiante favoreció la iniciativa y enriqueció el aprendizaje individual.

Con toda esta experiencia, puedo afirmar que mi labor docente se ha modificado y enriquecido; aún no logro dominar todos los recursos, cada clase me retroalimenta, pero puedo asegurar que el proceso pedagógico ya no es el mismo, García comenta al respecto

En este nuevo escenario el profesor debe modificar su rol en el proceso de aprendizaje, convirtiéndose en el organizador de la interacción entre los alumnos y los objetos de conocimiento, en el generador de interrogantes, estimulando permanentemente a los alumnos en la iniciativa y en el aprendizaje activo con creación, comunicación y participación. Debe guiar los procesos de búsqueda, análisis, selección, interpretación, síntesis y difusión de la información. (García 2010, p. 5)

En síntesis, las TIC utilizadas como recurso en los cursos, fueron las siguientes

14 Disponible en http://drogasmitosyrealidades.blogspot.com 


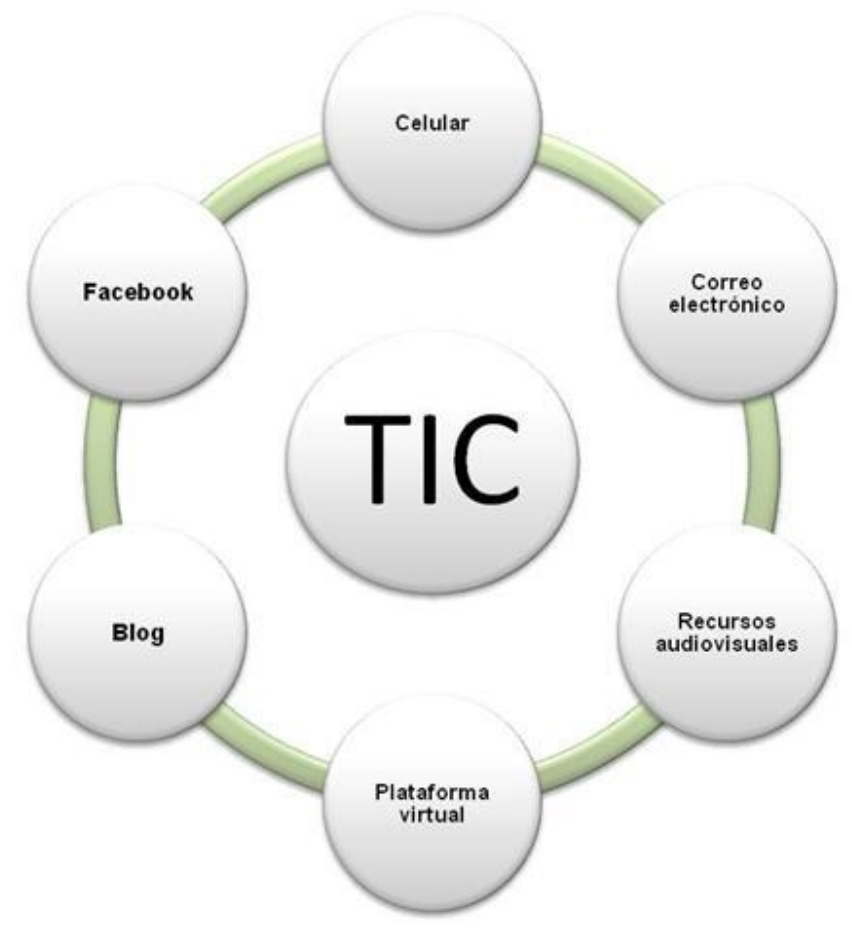

Cada uno de estos recursos tecnológicos ha tenido un uso específico que se detalla a continuación:

\section{Esquema de trabajo utilizando las TIC durante los cursos de la carrera Trabajo Social}

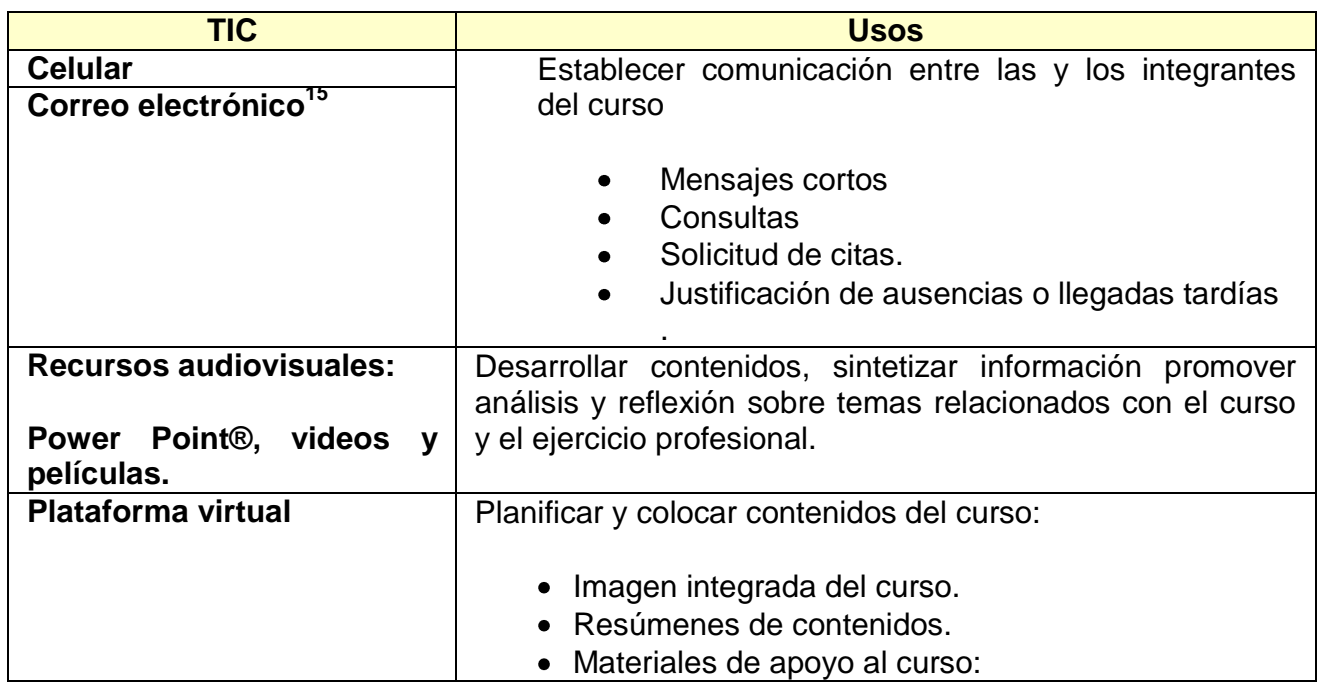

15 Los mensajes de texto por celular han provocado una disminución en el uso del correo electrónico, ya que el celular es un medio de comunicación móvil, que cargan de manera permanente y les avisa en tiempo real el ingreso de un mensaje. 


\begin{tabular}{|c|c|}
\hline & $\begin{array}{l}\text { - Presentaciones } \\
\text { - Producción estudiantil. } \\
\text { - Videos } \\
\text { - Seguimiento a la utilización de recursos por parte } \\
\text { de cada estudiante } \\
\text { Comunicación ágil. } \\
\text { - Envío de mensajes a la población estudiantil } \\
\quad \text { matriculada en el curso. } \\
\text { Acceso a recursos } \\
\text { Foros de discusión sobre contenidos del curso. } \\
\text { Tareas. }\end{array}$ \\
\hline Facebook® & $\begin{array}{l}\text { Espacio para consultas y construcción de una bitácora con } \\
\text { preguntas y respuesta: }{ }^{16} \\
\text { - Es un espacio abierto } \\
\text { - Comunicación informal } \\
\text { - Tutorías cortas } \\
\text { - Consultas sobre tareas } \\
\text { Acceso a la dinámica y realidad de la población estudiantil } \\
\text { al ingresar a los diferentes perfiles. }\end{array}$ \\
\hline Blog & $\begin{array}{l}\text { Espacio informal para elaborar comentarios sobre los } \\
\text { diferentes materiales que se colocan: noticias, lecturas, } \\
\text { videos u otros relacionados con el curso. } \\
\text { Colocar material novedoso diferente a las lecturas } \\
\text { obligatorias del curso que amplían los contenidos y } \\
\text { favorecen la discusión. }\end{array}$ \\
\hline
\end{tabular}

El uso de las TIC en el proceso pedagógico permite incorporar un modelo flexible de interacción entre el equipo integrado por el docente y la población estudiantil, facilita el acompañamiento particular y general del proceso de aprendizaje de la población estudiantil, así como el desarrollo de habilidades y destrezas, tanto de la docente como de la población estudiantil, y fomentar el protagonismo de la población estudiantil en la decisión de utilizar las herramientas, guías de estudio, ejercicios y contenidos que se ponen a su disposición por medio de herramientas concretas como el blog, los foros, las presentaciones en Power Point $\AA_{\text {, }}$ enlaces a otros espacios virtuales, etc., que además le permiten desarrollar la curiosidad, la autodisciplina, resolver situaciones por sí mismos y continuar construyendo su propio aprendizaje

16 Se abrió un perfil específico para dar respuestas a las consultas de la población estudiantil matriculada, así que solo esa población tiene acceso a escribir en ese espacio. 
De esta manera, se motiva a la población estudiantil a construir su propio conocimiento, a buscar el significado que ello tiene para cada uno o cada una, procesarlo y transmitirlo desde su comprensión y desarrollar habilidades para la aplicación de este conocimiento en su campo de formación profesional. Por lo tanto, los recursos virtuales dan la oportunidad de trascender el espacio del aula presencial y fomentar el avance individual ampliando los contenidos propios del curso, pues estimula la búsqueda de más información, siempre el estudiante es quien tiene el protagonismo y toma la decisión sobre hasta dónde llegar.

\section{La respuesta de la población estudiantil al uso de las $\mathrm{TIC}^{17}$}

En el momento en el que inicié las primeras experiencias utilizando las TIC, tuve como premisa que la población estudiantil tiene dominio de la computadora como herramienta, usan la Internet y tienen habilidades y destrezas en la búsqueda de información y enfrentan con facilidad el uso de nuevos software, y las nuevas tecnologías son parte de su ambiente natural donde se han desenvuelto en su proceso educativo. Todo ello permitiría tener una experiencia novedosa.

Sin embargo, no podía ocultar mi preocupación, porque era yo la que tenía menos habilidades y destrezas en el uso de las TIC, y en el entorno educativo mantenemos la premisa de que el profesor es el que más sabe.

Una de las primeras impresiones fue que la población estudiantil en su mayoría no ingresa a los recursos por iniciativa propia, lo hacen cuando es parte de las obligaciones del curso. En mi primera experiencia durante las tres primeras semanas de clase solamente el 50\% de la población estudiantil ingresó al "aula virtual" para revisar los contenidos o tareas pendientes, buscaban otros medios para saber sobre las asignaciones, consultaban al resto de la población matriculada en el curso o fotocopiaban los materiales que otros habían bajado de la plataforma virtual. El $100 \%$ de la población ingresa cuando debe realizar una acción concreta que es evaluada desde el aula virtual como los foros o las tareas.

Para orientar la labor de la docente, se aplicó al inicio de cada curso una encuesta sobre tenencia de computadoras y acceso a Internet, a la población estudiantil que se matriculó en los

\footnotetext{
17 La información que se presenta fue aportada por la población estudiantil de la Escuela de Trabajo Social de la Universidad de Costa Rica, matriculada en los cursos TS-5118: Ideología Ética y Derechos Humanos III, I ciclo lectivo 2010 y TS. 4018: Fundamentos teórico metodológicos del trabajo social VI: Fenómeno de las drogas, II ciclo lectivo 2010.
} 
cursos impartidos con apoyo de las TIC: TS-5118: Ideología Ética y Derechos Humanos III, I ciclo lectivo 2010 y TS. 4018: Fundamentos teórico metodológicos del trabajo social VI: Fenómeno de las drogas, II ciclo lectivo 2010. Antes de finalizar el ciclo lectivo se aplicó una encuesta de opinión a la misma población estudiantil de los dos cursos, con preguntas abiertas.

La población estudiantil considera ${ }^{18}$ que las TIC facilitan el proceso de aprendizaje, identifican aportes de cada uno de los recursos utilizados como se puede apreciar a continuación.

\section{Facebook}

La población estudiantil considera que esta herramienta: "acelera la comunicación casi inmediata y se comparte con varios" y "es una red social muy accesible y para fines educativos está bien. Así es más fácil comunicarse con la profe". Sobre los usos manifiestan que: "es informativo, práctico", "es entretenido. Atractivo", "me gusta son actuales y de fácil ingreso".

La evalúan como una: "buena herramienta para hacer consultas", "un buen espacio para hacer algún comentario", "me parece que es una buena manera de hacer consultas sobre los trabajos", "me parece útil, pero primero reviso las indicaciones en otras herramientas"

También existen estudiantes que no están de acuerdo con el uso de esta herramienta como recurso, justifican su posición con los siguientes comentarios: "personalmente no me gusta para trabajos o consultas, prefiero el correo de hecho antes estaba en contra de esas redes porque todo el mundo ve", otro comentario en esta línea: "personalmente ha facilitado mucho la comunicación sin embargo no me ha parecido útil para el curso"

Las sugerencias de la población estudiantil hacia el uso de este recurso se pueden ubicar en dos posiciones unas orientadas a utilizarla más: "Aprovechar más este espacio en tanto es ahí donde hay más conocimiento del uso por parte de las y los estudiantes" y otras sugieren "valorar si es realmente pertinente".

\section{Aula virtual}

La población estudiantil se refiere al aula virtual como: "un buen espacio donde no solo tengo acceso a las fechas y las entregas de trabajo sino que también a diferentes materiales

18 En este artículo se transcriben las opiniones de la población estudiantil tal y como respondió. 
(por ejemplo mapas conceptuales)", "es la que más utilizo muy completa fácil de usar, la información está ordenada y es fácil ver las tareas pendientes y compartir documentos", "permite colocar la clase más allá del aula".

De los recursos utilizados el aula virtual es la que: "más les gusta”, justifican su respuesta indicando que: "muy completo y accesible", "más actual y de fácil ingreso", "es sencillo y accesible más para la gente como yo que tenemos horario muy largos y no podemos venir a todas las clases". La evalúan de manera positiva y rescatan los siguientes elementos: "es excelente para ubicarse en el curso y repasar lo visto en clase me agrada", "excelente herramienta. Me parece muy bien que ahí se puedan colocar resúmenes notas etc. Al momento de realizar un trabajo se tienen a la mano todos los materiales", "importante para acceder a los documentos y trabajos de las lecturas. Además que envía información por correo", "Es una buen herramienta que facilita el acceso a trabajo, presentaciones, indicaciones entre otros".

Por su parte, existen estudiantes que tienen una opinión negativa como: "no me parece tan útil, no interactuamos como grupo, es más de uso personal, hasta ahora solo para revisar indicaciones y lecturas" $y$ "no me parece tan fácil de navegar con frecuencia me pierdo".

Sugiere la población estudiantil "continuar con su uso". En relación con la colocación de materiales en el aula virtual hay posiciones encontradas, algunos señalan: "facilitar lecturas $y$ presentaciones solo por esta vía", mientras que otra parte indica que: "las lecturas estén también en fotocopiadora, yo no tengo impresora y me es más económico fotocopiarlas que imprimirla".

\section{Blog}

A la población estudiantil este recurso les pareció novedoso e interesante: "muy útil me parece la mejor herramienta", y a diferencia de los otros recursos todas las posiciones fueron positivas: "muy importante y actual", "buena porque todos participamos", "genera debate. Buena herramienta", "interesante porque comparte opiniones de un determinado tema". "muy buena herramienta nos permite plantear nuestra opinión crítica, impresión, además de debatir a partir de la opinión de las otras (os) compañeras(os)", "me gusta mucho comentar y leer los comentarios de las (os) compañeras (os)" 
Evidencian las opiniones de la población estudiantil que es un recurso que algunos desconocían o nunca habían utilizado: "es novedosa pero por falta de costumbre a veces se dificulta un poco usarla".

Entre las sugerencias que plantea la población estudiantil destaca la insistencia de "invitar a más gente a participar" y colocar materiales de lectura cortos "sin embargo la lectura es agotadora por la densidad de los textos".

Llama la atención la sugerencia que plantea "avisar por otro medio cuando hay cosas nuevas en el blogger", esta petición evidencia el desconocimiento y poco uso del recurso, ya que el mismo software puede informar a sus seguidores, a la dirección electrónica que anotaron al inscribirse, cada vez que hay alguna novedad en el blog.

\section{Correo electrónico}

El correo electrónico es el medio que más conoce y utiliza la población estudiantil como se puede apreciar en los siguientes comentarios: "es la que más utiliza, la más rápida y fácil de saber que tareas hay pendientes", "muy importante y actual", "ágil, rápida útil". Lo utiliza para: "intercambiar mensajes, archivos además facilita la comunicación", "consultas breves sin embargo las otras herramientas han hecho que no haya tenido la necesidad de utilizarla".

La población estudiantil siente cercanía y seguridad con este medio lo que se puede apreciar con las siguientes impresiones: "espacio más personalizado para hacer comentarios y consultas", "yo prefiero que manden las lecturas por correo que por el blog y el aula virtual porque es más sencillo", "mandar todos los materiales que se suben al blog y al aula virtual también por correo así de fijo y de cualquier forma la tenemos". La principal sugerencia fue: "continuar con su uso"

Destaca de manera insistente como limitación para el uso de estos recursos, por parte de la población estudiantil matriculada en los dos cursos en estudio, el no contar con computadora y/o acceso a la web en sus hogares, actividad que realizan por lo general en los laboratorios de cómputo con que cuenta la Universidad, de ahí que sugieran: "no poner cosas entre sábado y domingo preferiblemente". 
Al respecto, en la encuesta ${ }^{19}$ sobre la tenencia de computadoras y el acceso que tienen a la web desde sus hogares, los resultados demuestran que entre un $5 \%$ y un $15 \%$ de la población estudiantil matriculada no tiene computadora en el hogar y entre un $20 \%$ a $28 \%{ }^{20}$ no tiene acceso a la Internet (se incluye en estos porcentajes la población que no tienen computadora). Esta situación está relacionada directamente con las condiciones socioeconómicas de la población estudiantil encuestada, ya que un $85 \%$ en el I ciclo lectivo y un 92\% en el II ciclo lectivo 2010 del total de la población estudiantil matriculada en los cursos en estudio disfrutaron de beca por condición socioeconómica, lo que evidencia que proceden de familias con limitados recursos económicos.

A pesar de que tener acceso a laboratorios de cómputo conectados a la Internet tanto en la Unidad Académica como en la Universidad en general, realmente no es lo mismo el tiempo que pueden dedicar a estar "conectados". Esta situación es evidenciada ${ }^{21}$ en el uso de los recursos, mientras que un 55\% revisa el correo varias veces al día, un 35\% lo hacen entre 1 o 4 veces a la semana y el otro $10 \%$ refiere que revisa el correo una vez al día. También sucede a pesar de que la población estudiantil planteó que es el recurso que más utilizan. Por su parte, un $10 \%$ solo tiene una dirección para recibir correos y un $65 \%$ tiene dos y el restante $25 \%$ más de dos direcciones electrónicas.

Por lo tanto, entre la población estudiantil existe una diferenciación importante en las posibilidades de acceso a las TIC, que está determinado por la extracción social de la población. Tener o no acceso a computadora con internet desde el hogar y además de que la conexión "sea buena" (que les permita navegar sin que los esté cerrando la página) favorece el desarrollo de habilidades y destrezas en el uso de herramientas en la web, así como el acceso a la información y el conocimiento. La población que no cuenta con este recurso debe buscar sitios de acceso en la Universidad que tiene un horario definido y un cupo específico, en otros casos, cuando tienen los recursos económicos, pagar el uso de estos recursos, en los llamados "cafés internet", ambientes que no necesariamente favorecen el desarrollo académico de la población estudiantil.

\footnotetext{
19 Se aplicó una encuesta a la población estudiantil de la Escuela de Trabajo Social de la Universidad de Costa Rica, matriculada en los cursos TS-5118: Ideología Ética y Derechos Humanos III, I ciclo lectivo 2010 y TS. 4018 : Fundamentos teórico metodológicos del trabajo social VI: Fenómeno de las drogas, II ciclo lectivo 2010.

${ }^{20}$ La encuesta se aplicó a población estudiantil de dos cursos diferentes de ahí que se presentes dos datos diferentes.
} 
Esta información acercó a la docente a la realidad de la población estudiantil y por lo tanto, le señala los retos en el curso, para incorporar el uso de las TIC. Por una parte, la población estudiantil no tiene el mismo conocimiento ni desarrollo de habilidades y destrezas en el uso de las TIC, por lo que debe hacer inducción sobre los recursos que van a utilizar durante el curso, para favorecer la motivación de la población estudiantil, ya que el curso les ofrece, además de los contenidos propuestos curricularmente, el desarrollo de habilidades y destrezas en uso de las TIC como herramientas de trabajo como estudiantes y futuros profesionales.

Por otra parte, no contar con acceso a la Internet desde los hogares limita la posibilidad de realizar actividades, como por ejemplo, los foros en tiempo real, y mantener comunicación con toda la población estudiantil en horario nocturno y fines de semana, consideraciones necesarias en la planificación de las actividades del curso.

\section{A modo de cierre}

La sociedad ha logrado un desarrollo tecnológico que se ha introducido tanto en el entorno económico y laboral como en el ámbito educativo y familiar, que ha provocado cambios en las relaciones económicas y sociales.

Las Tecnologías de la Información y de la Comunicación han logrado trascender el espacio y el tiempo en la comunicación y en la disponibilidad de información y conocimiento, el cual se encuentra al alcance de todos en la web. Sin embargo, el acceso a la tecnología no es el mismo para toda la población, ya que los sectores sociales con limitados recursos económicos tienen menor acceso a las TIC.

Las generaciones más jóvenes y jóvenes adultos al nacer en una época de mayor avance tecnológico poseen habilidades y destrezas para el uso de las TIC que no tienen las generaciones adultas.

Por tal motivo, las nuevas generaciones demandan en los procesos educativos el uso de los recursos tecnológicos que ya han incorporado como parte de su vida diaria. Empero, la población docente se enfrenta a una población estudiantil que no tiene el mismo acceso a las TIC y, por lo tanto, tampoco tiene el mismo desarrollo de habilidades y destrezas en su uso.

21 Resultado de la encuesta aplicada a la población estudiantil de Trabajo Social matriculada en los dos cursos en estudio. 
Para la población docente el uso de las TIC se convierte en un gran reto, ya que debe vencer las barreras personales que tiene hacia este recurso, desarrollar habilidades y destrezas en el uso de las TIC, trabajar con una población con diferente acceso, así como diversidad en el desarrollo de habilidades y destrezas.

En ese sentido, las nuevas tecnologías requieren de la construcción de un proceso pedagógico flexible que fortalezca el protagonismo de la población estudiantil con el debido acompañamiento de la población docente desde otras estrategias que permitan el desarrollo de capacidades: de investigación, análisis, crítica y autocrítica, selección, organización y construcción de conocimiento entre otras.

Asimismo, el uso de las TIC modifica la relación tradicional entre el o la docente y la población estudiantil, pues la interacción trasciende el espacio físico del aula y las horas tradicionales de atención presencial, a espacios abiertos, en tiempo real y tiempo diferido, con flexibilidad tanto de horario como en la interacción y en la emisión de la comunicación que se genera con la participación de dos o más personas, sin que deba planearse con antelación. Todo ello requiere de la población docente horas de dedicación a estos nuevos espacios.

De igual modo, la familiaridad de las TIC para la población estudiantil va a estar determinada por el acceso que tengan, fundamentalmente desde sus hogares, ya que se han convertido en herramientas de uso cotidiano y permanente, mientras elaboran un trabajo para la universidad, buscan artículos y libros, conversan con sus amigos y compañeros, se enteran de noticias, observan mensajes entre sus amigos y amigas y las y los amigos y amigas de sus amigos y amigas, jah! y también entre sus familiares, se intercambian materiales y música, reproducen videos entre otros.

Por su parte, la población estudiantil que debe utilizar las TIC en laboratorios de la Universidad o lugares comerciales, dedican menor tiempo a estos espacios y concentran su atención en cumplir con las tareas de los cursos, más que en lograr toda la riqueza de interacción que favorecen las TIC.

Estas son las razones de encontrarse con diferentes posiciones frente al uso de las TIC en las experiencias educativas compartidas, mientras que una parte de la población estudiantil plantea que se debe continuar con el uso de las TIC en el proceso educativo, otra parte que se debe valorar su pertinencia, una parte de la población desea que las lecturas sean impresas otra parte que sea digital y esto como una consecuencia entre otras, de la inequidad en el acceso a las TIC. 
A pesar de los esfuerzos que la Universidad de Costa Rica ha realizado para lograr el acceso y utilización de las TIC aún tiene grandes retos como los siguientes:

- Mejorar la conectividad en los edificios.

- Mayor disposición de equipo para docentes y estudiantes.

- Programas de apoyo y asesoría a la población estudiantil sobre las TIC.

- Programas de asesoría y apoyo permanentes a la población docente.

Asimismo, la población docente por su parte debe realizar esfuerzos para:

- Conocer las características de la población estudiantil.

- Implementar procesos educativos flexibles centrados en el estudiante incorporando las TIC como recurso pedagógico.

- Continuar con el desarrollo de habilidades y destrezas en el uso de las TIC.

- Crear nuevos experiencias pedagógicas que favorezcan en la población estudiantil la curiosidad, la autodisciplina, el resolver situaciones por sí mismos y continuar construyendo su propio aprendizaje.

\section{Referencias}

Area, Manuel. (2009) Introducción a la Tecnología Educativa. Recuperado el 20 de noviembre 2010 de http://webpages.ull.es/users/manarea/ebookte.pdf

Bustos González Atilio. (s.f.). Estrategias didácticas para el uso de las TIC's en la docencia universitaria presencial. Un manual para los ciudadanos del Ágora. Recuperado el 8 de marzo 2011 de http://books.google.com/books?id=ngxq2nYRD1gC\&pg=PA38\&dq=tics+en+la+educacio n\&hl=es\&ei=taN2Tf7zD4LSsAPjzMS0BA\&sa=X\&oi=book result\&ct=result\&resnum=6\&v ed=0CEUQ6AEwBQ\#v=onepage \&q\&f=false

Mato, María A., Santamaría, Julio, Silva, José de S., Cheaz, Juan. (2001). La dimensión de gestión en la construcción de la sostenibilidad institucional (Serie Innovación para la innovación Institucional). San José, C.R.: Proyecto ISNAR "Nuevo Paradigma"

García, Felipe. (2010). Nativos digitales y modelos de aprendizaje. Recuperado el 22 de julio 2010 de http://spdece07.ehu.es/actas/Garcia.pdf

Programa de la Sociedad de la Información y el Conocimiento de la Universidad de Costa Rica. (2006). Hacia la Sociedad de la información y el conocimiento: Informe 2006. San José: PROSIC. 
Programa de la Sociedad de la Información y el Conocimiento de la Universidad de Costa Rica. (2008a). Hacia la Sociedad de la información y el conocimiento: Informe 2007. San José: PROSIC.

Programa de la Sociedad de la Información y el Conocimiento de la Universidad de Costa Rica. (2008b). Los jóvenes costarricenses en la Sociedad de la Información y el Conocimiento. San José: PROSIC.

Universidad de Costa Rica. (1974). Estatuto Orgánico de la Universidad de Costa Rica. San José: La Universidad.

Universidad de Costa Rica. (2008, 21 de noviembre). Políticas de la Universidad de Costa Rica para los años 2010-2014. Gaceta Universitaria 40-2008. San José: La Universidad.

Universidad de Costa Rica. (2010a). Directrices Generales en Tecnologías de Información y comunicación (TIC's) para la Universidad de Costa Rica. u-digit@I. Plan de mejora 2010. San José: La Universidad.

Universidad de Costa Rica. (2010b). Estadísticas I y II ciclo 2009. Vicerrectoría de Vida Estudiantil-Oficina de Becas y Atención Socioeconómica (Publicación interna). San José: Universidad de Costa Rica.

Tünnermann, Carlos. (1999). Educación Superior de cara al Siglo XXI. San José: Editorial Mirambell. 